\title{
Poisson statistics of sparkling water bubbles
}

\author{
Stojan Rendevski ${ }^{1}$ and Riste Popeski-Dimovski ${ }^{2}$ \\ ${ }^{1}$ Faculty of Engineering and Technology, Department of Mathematics and Natural Sciences, Higher Colleges of \\ Technology, Ras al Khaimah Men's Campus, 4793 Ras al Khaimah, United Arab Emirates \\ ${ }^{2}$ Institute of Physics, Faculty of Natural Sciences and Mathematics, University "Ss. Cyril and Methodius", \\ Skopje, Republic of Macedonia
}

\begin{abstract}
A simple acoustic measurement method has been applied to demonstrate Poisson distribution of bubbles sparkling at a water surface. The quality of sparkling water has been determined by quantifying the characteristic time of decay of number of air bubbles sparkling at the surface of water served to consumers. The average sound frequency of the bubbles at sparkling has been found dependent on the chemical composition of the water (carbon dioxide and minerals concentration). The acoustic measurement method proved to be fast and reliable for determining the quality of appearance of served sparkling water.
\end{abstract}

Keywords: Poisson distribution, Acoustic signal processing, Bubble dynamics

\section{Introduction}

The good quality of sparkling water served to consumers can be seen in respect of prolonged effect of air/carbon dioxide bubbles emerging to surface thus giving a feeling of water freshness while drinking. Another aspect of good quality of sparkling water is bringing the same freshness feeling to consumers while limiting the carbon dioxide content in the water at lower level. The feeling of sparkling bubbles while drinking experienced by tickling the lips, tongue and nostrils depends mostly on the mineral content of the water, the type of the serving glass and the serving temperature [1]. The imaging technique is hard to implement for testing because of the complexity of air and carbon dioxide bubbles mixture emerging at different size, speed and at different pressure at the water surface (the sparkling moment). One reliable and fast method for testing the time extension of the sparkling effect of the water is proposed below based on sound measurement of the sparkling bubbles. This method of acoustic analysis of the sparkling bubbles gives also good correlation with the concentration of the carbon dioxide and concentration of the minerals, especially the carbonated minerals. Precisely speaking, there is a direct relationship between the number of sparkling bubbles (sound intensity) in a given time interval and the chemical composition of the sparkling water.

\section{Methods And Experiments}

The experimental method is very simple (Fig.1). Opened bottle of sparkling water is positioned on a table without vibrations and a microphone ( $300 \Omega$ impedance) is placed through the bottle neck $1 \mathrm{~cm}$ above the water surface. The microphone from a multimedia set of headphone/microphone can serve for the purpose. The microphone is connected to a PC with standard sound card (Fig.2).

GoldWave software (Gold Wave Inc., Canada) has been used for sound recording with sampling rate of 44,100 Hz (Fig.3a). The experiments have been conducted with two different sparkling water samples: type SW1 with normal concentration of carbon dioxide and minerals and type SW2 with lower content. Fourier analysis of the power spectra of the sound at sparkling has been applied. The Fourier transform of the amplitude-time sound signal (Fig.3b) to amplitude-frequency function has been done for the two types of water samples from where the characteristic frequency of the sparkling bubbles sound was easily found (at the peak, Fig.3c).

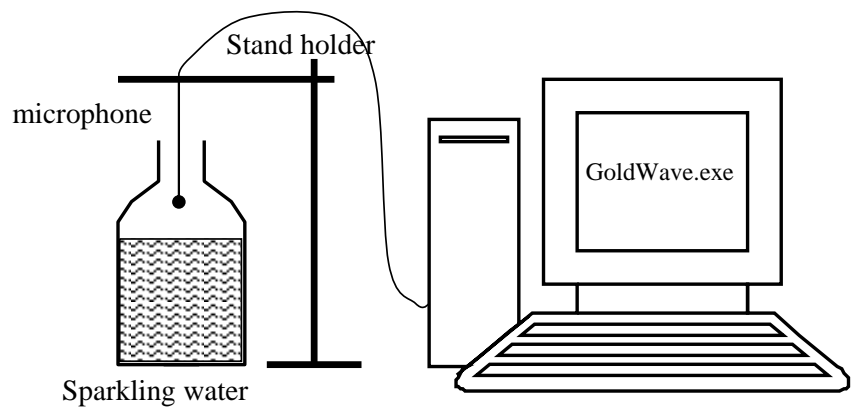

Figure 1. A scheme of the experimental set-up. 


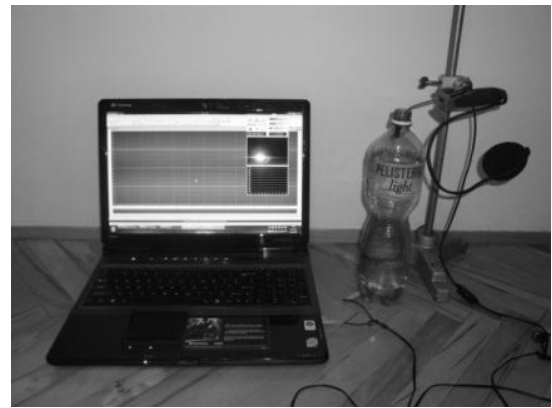

Figure 2. A photo of the experimental set-up.

One example of the recorded amplitude-time dependence sound signal for bubbles sparkling at water surface is shown on figure $3 \mathrm{~b}$. The figure represents the amplitude-time function of the sound wave emerged from one single bubble at sparkling. After Fourier transform has been done by using the OriginPro software (Origin Lab Corp., USA), the distribution of the sound intensity on the frequency is obtained (Fig.3c).

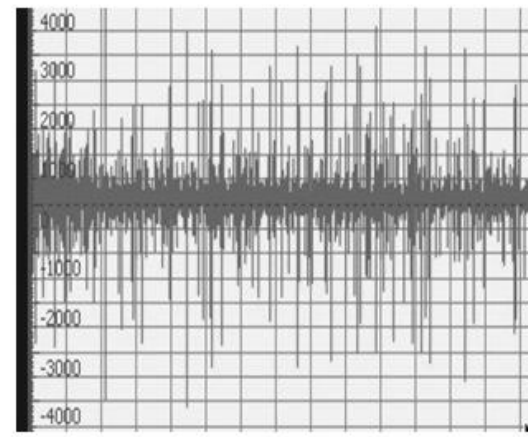

Fig.3a. Recorded sound (many bubbles).

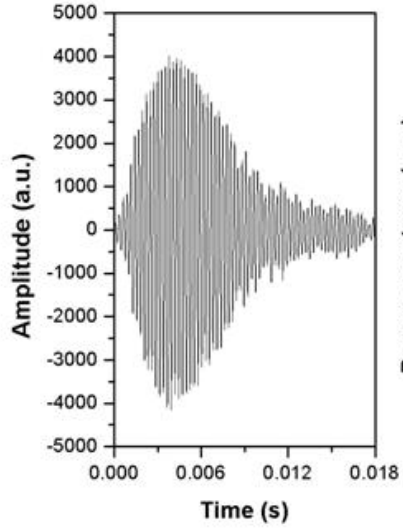

Fig.3b. Recorded sound of a single bubble (extracted from fig.3a).

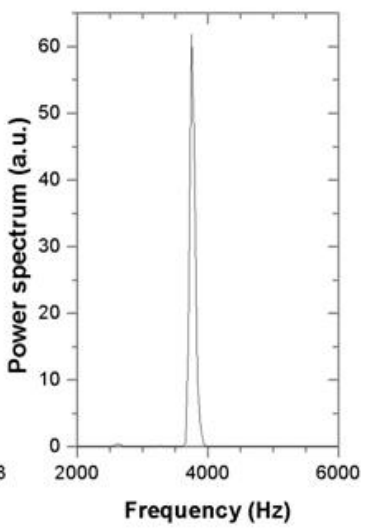

Fig.3c. Fourier transform on signal from fig. $3 b$.

\section{Discussions And Conclusions}

In the experiments, sparkling events with same sound intensity (amplitude) have been counted in wide time interval. An exponential decay of the number of sparkling has been observed. After interpolation of the experimental data to an exponential function, the so called characteristic time $\tau$ of the bubbles decay has been determined (Fig.4a and Fig.4b). This parameter $\tau$ can be seen as a measure for the quality of sparkling water appearance. Namely, for water for which the $\tau$ parameter is large (Fig.4a), the water will have prolonged sparkling appearance when served. This is wanted quality of the sparkling water for most of the consumers.

When a glass of sparkling water is served, air/carbon dioxide bubbles popping-up at the surface can be observed with some probability that seems same as for the radionuclide decay. For the radionuclide decay, it has been found long time ago that these incidental events are following the Poisson statistics [2]. In order to prove that sparkling occurs according to the Poisson statistics, the popping sound with same intensity have been recorded in relatively long time interval of several minutes. Here one must be careful. If we are going to make an analogy with the radionuclide decay of same type of nuclides (atoms), this must be identified with same type of sparkling bubbles - bubbles with same dimension and pressure that will produce sound with same intensity, for example, in the range of 250 a.u. to 300 a.u. 


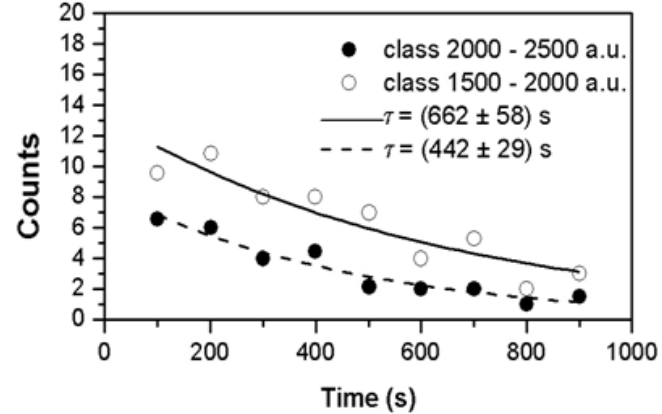

Figure 4a. Water type SW1.

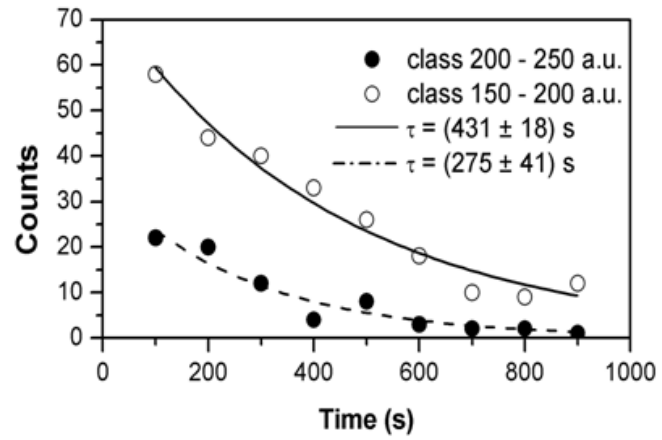

Figure 4b. Water type SW2.

The Poisson distribution of events mathematically can be expressed with the formula:

$$
P(n)=\frac{\lambda^{n} e^{-n}}{n !},
$$

where $\lambda$ is the average value of the events distribution and $n$ is the number of the observed events (sparkling or popping-up of bubbles). On Fig.5, the experimental cumulative distribution of the events is given (with circles). On the same figure, the theoretical cumulative Poisson distribution of the events is given (with full line), also. A good fit of the experimental data to the theoretical function of the Poisson distribution can be noticed. On the same graph, the Gaussian cumulative distribution of the events is given only for comparison. The Gaussian distribution is different from the Poisson distribution because it is useful for modeling events that arise in so called binomial experiments (one outcome out of two possible) while the Poisson distribution is useful for modeling events that take place in a haphazard way (one event in no connections with any other).

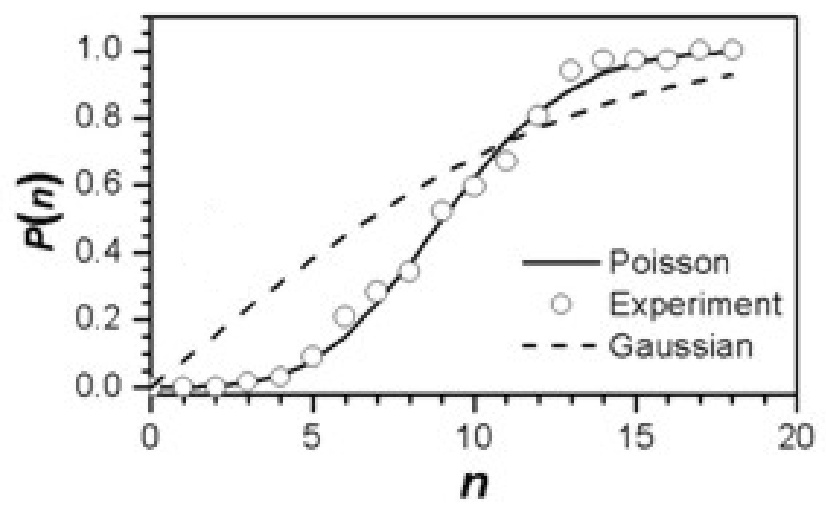

Figure 5. Experimental cumulative distribution of events with the Gaussian and Poisson theoretical cumulative distributions.

Once the Poisson distribution has been proved valid for statistical analysis of the experimental data, the characteristic time of decay $\tau$ of the number of sparkling bubbles can be obtained easily. Here, an analogy can be made between the half-lifetime of radionuclides and the characteristic time $\tau$ of sparkling bubbles - time for which the number of sparkling bubbles decreases for $e$-times after serving the sparkling water in a glass [2]:

$$
N=N_{0} \cdot e^{-\frac{t}{\tau}}
$$

where $N_{0}$ - number of popping-up bubbles at zero time (water serving moment) and $N$ - number of popping-up bubbles at time $t$ after serving. The calculated parameter $\tau$ is the quantity we need for testing the quality of appearance of sparkling water. Higher value means prolonged effect of sparkling. 
Table 1. Sound frequency for bubbles sparkling with different intensity

\begin{tabular}{|c|c|c|}
\hline $\begin{array}{l}\text { Sound intensity } \\
\text { (a.u.) }\end{array}$ & $\begin{array}{cc}\text { I. } & \text { FREQUENCY }(\mathrm{Hz}), \\
\text { II. } & \text { WATER TYPE SW1 }\end{array}$ & $\begin{array}{ll}\text { III. } & \text { FREQUENCY }(\mathrm{Hz}) \text {, } \\
\text { IV. } & \text { WATER TYPE SW }\end{array}$ \\
\hline $2000-2500$ & $3780 \pm 180$ & not existed \\
\hline $1500-2000$ & $3230 \pm 90$ & not existed \\
\hline $1000-1500$ & $2510 \pm 90$ & $1240 \pm 60$ \\
\hline $500-1000$ & $1170 \pm 70$ & $443 \pm 50$ \\
\hline $200-500$ & $485 \pm 25$ & $218 \pm 20$ \\
\hline
\end{tabular}

Fourier transform of the recorded sound signals has been performed. A work has been done on several chosen single bubbles popping-up with different sound intensity taken from sound recordings that lasted up to 15 minutes. It has been found that the sparkling happens with sound in narrow frequent region (Fig. 3c). In Table 1 , the average frequency of the sound is given for the two different types of water (SW1 and SW2) and for different size of bubbles (different sound intensity). The sound frequency from the larger bubbles of water type SW2 is not shown in table 1 because there were no large bubbles in that type of water that can give enough sound intensity at sparkling that can be recorded by the microphone. This is expected for the so called "light" sparkling water because of the small concentration of minerals and carbon dioxide. It has been found from the experiments that large differences in average sound frequency (Table 1) exist because of the large differences of the chemical composition of the bottled waters. Namely, it is well known that the sound frequency at sparkling depends on the elastic properties of the thin water membrane around the bubbles at the moment of emerging on the water surface [3]. The different chemical composition of the two sparkling water types and the differences of the carbon dioxide partial gas pressure inside the bubbles have influence on the elastic properties of the thin water membrane around the bubbles.

Another interesting finding has arisen from the experiments. In several occasions, when large bubbles sparkled at the water surface, a sparkling of other smaller bubbles has been induced (triggered), which cannot occur at given time if there was no large bubble sparkling at that moment. These kinds of events can be examined by using the Markovian statistics. The Poisson events are statistically independent of each other. That means that sparkling of one bubble at given moment of time does not cause premature or delayed sparkling of another bubbles. Unlike the Poisson events distribution, the Markovian events are interdependent with determined probability. In our case, that was manifested by premature sparkling at same moment of 2-3 small bubbles when one much larger bubble "exploded".

The research on gas bubbles acoustics can be of great usefulness. In brewery industry, for example, during fermentation in the bioreactors, a large amount of gas bubbles is released. Such bubbles bring information how the brewing process is running. Other researchers can propose more miscellaneous examples of practical usefulness of knowing the properties of gas bubbles.

\section{References}

[1]. E. K. Rogers. Instrumentation \& Sensors for the Food Industry (Boca Raton, USA: CRC Press, 1998)

[2]. D. Halliday, R. Resnick, J. Walker, Fundamentals of Physics, 7th Edition (New York, USA: John Wiley and Sons, 2005)

[3]. $\quad$ R. Clift, J.R. Grace, M.E. Weber, Bubbles, Drops and Particles (New York, USA: Academic Press, 1978) 